\title{
Ball convergence for Steffensen-type fourth-order methods
}

\author{
Ioannis K. Argyros and Santhosh George \\ Cameron University and NIT Karnataka
}

\begin{abstract}
We present a local convergence analysis for a family of Steffensen-type fourth-order methods in order to approximate a solution of a nonlinear equation. We use hypotheses up to the first derivative in contrast to earlier studies such as [1], [5]-[28] using hypotheses up to the fifth derivative. This way the applicability of these methods is extended under weaker hypotheses. Moreover the radius of convergence and computable error bounds on the distances involved are also given in this study. Numerical examples are also presented in this study.
\end{abstract}

Keywords - Newton method, Steffensen-type methods, order of convergence, local convergence.

\section{INTRODUCTION} $\mathrm{N}$ this study we are concerned with the problem of approximating a
locally unique solution $x^{*}$ of equation

$F(x)=0$,

where $F: D \subseteq S \rightarrow S$ is a nonlinear function, $D$ is a convex subset of $S$ and $S$ is $\mathbb{R}$ or $\mathbb{C}$. Artificial intelligence and e-learning are two of the emerging needs of the information age. Authors from various other areas can follow these techniques to serve another scientific communities. Newton-like methods are famous for finding solution of (1), these methods are usually studied based on: semi-local and local convergence. The semi-local convergence matter is, based on the information around an initial point, to give conditions ensuring the convergence of the iterative procedure; while the local one is, based on the information around a solution, to find estimates of the radii of convergence balls $[3,4,20,21,22,24,26]$.

Third order methods such as Euler's, Halley's, super Halley's, Chebyshev's [1]-[28] require the evaluation of the second derivative $F^{\prime \prime}$ at each step, which in general is very expensive. That is why many authors have used higher order multipoint methods [1]-[28]. In this paper, we study the local convergence of fourth order Steffensen-type method defined for each $n=0,1,2, \cdots$ by

$$
\begin{aligned}
& y_{n}=x_{n}-\frac{2 F\left(x_{n}\right)^{2}}{F\left(x_{n}+F\left(x_{n}\right)\right)-F\left(x_{n}-F\left(x_{n}\right)\right)} \\
& x_{n+1}=x_{n}-\frac{2 F\left(x_{n}\right)^{2}}{F\left(x_{n}+F\left(x_{n}\right)\right)-F\left(x_{n}-F\left(x_{n}\right)\right)} \frac{F\left(y_{n}\right)-F\left(x_{n}\right)}{2 F\left(y_{n}\right)-F\left(x_{n}\right)},
\end{aligned}
$$

where $x_{0}$ is an initial point. Method (2) was studied in [11] under hypotheses reaching upto the fifth derivative of function $F$.

Other single and multi-point methods can be found in [2, 3, 20, 25] and the references there in. The local convergence of the preceding methods has been shown under hypotheses up to the fifth derivative (or even higher). These hypotheses restrict the applicability of these methods. As a motivational example, let us define function $f$ on

$$
\begin{aligned}
D=\left[-\frac{1}{2}, \frac{5}{2}\right] \text { by } \\
\qquad f(x)=\left\{\begin{array}{l}
x^{3} \ln x^{2}+x^{5}-x^{4}, x \neq 0 \\
0, x=0
\end{array}\right.
\end{aligned}
$$

Choose $x^{*}=1$. We have that

$$
\begin{aligned}
& f^{\prime}(x)=3 x^{2} \ln x^{2}+5 x^{4}-4 x^{3}+2 x^{2}, f^{\prime}(1)=3, \\
& f^{\prime \prime}(x)=6 x \ln x^{2}+20 x^{3}-12 x^{2}+10 x \\
& f^{\prime \prime \prime}(x)=6 \ln x^{2}+60 x^{2}-24 x+22 .
\end{aligned}
$$

Then, obviously, function $f^{\prime \prime \prime}$ is unbounded on $D$. In the present paper we only use hypotheses on the first Fréchet derivative. This way we expand the applicability of method (2).

The rest of the paper is organized as follows: Section 2 contains the local convergence analysis of methods (2). The numerical examples are presented in the concluding Section 3.

\section{LOCAL CONVERGENCE FOR METHOD (2)}

We present the local convergence analysis of method (2) in this section. Let $U(v, \rho), \bar{U}(v, \rho)$ stand for the open and closed balls in $S$, respectively, with center $v \in S$ and of radius $\rho>0$.

Let $L_{0}>0, L>0, M_{0}>0, M>0$ and $\alpha>0$ be given parameters. It is convenient for the local convergence analysis of method(2) that

follows to define some function on the interval $\left[0, \frac{1}{L_{0}}\right)$ by

$$
g(t)=\frac{L t}{2\left(1-L_{0} t\right)}
$$

and parameters

$$
r_{A}=\frac{2}{2 L_{0}+L}<\frac{1}{L_{0}},
$$




$$
r_{0}=\frac{1}{\left(1+\frac{M}{2}\right) L_{0}}<\frac{1}{L_{0}} .
$$

Notice that if:

$$
M_{0} L_{0}<L \Rightarrow r_{A}<r_{0}
$$

$$
M_{0} L_{0}=L \Rightarrow r_{A}=r_{0}
$$

$$
M_{0} L_{0}>L \Rightarrow r_{0}<r_{A}
$$

We have that $g\left(r_{A}\right)=0$, and

$$
0 \leq g(t)<1 \text { foreach } t \in\left[0, r_{A}\right) .
$$

Define function $g_{1}$ on the interval $\left[0, r_{0}\right)$ by

$$
g_{1}(t)=\frac{L}{2\left(1-L_{0} t\right)}\left[1+\frac{2 \alpha M_{0} M^{2} t}{1-\left(1+\frac{M_{0}}{2}\right) L_{0} t}\right] t
$$

and set

$$
h_{1}(t)=g_{1}(t)-1
$$

We get that $h_{1}(0)=-1<0$ and $h_{1}(t) \rightarrow+\infty$ as $t \rightarrow r_{0}^{-}$. It follows from the Intermediate Value theorem that function $h_{1}$ has zeros in the interval $\left(0, r_{0}\right)$. Denote by $r_{1}$ the smallest such zero. Moreover, define function on the interval $\left[0, r_{0}\right)$ by

$$
p(t)=\left[L_{0} g_{1}^{2}(t)+\frac{4 M_{0} M}{1-\left(1+\frac{M_{0}}{2}\right) L_{0} t}+\frac{L_{0}}{2}\right] t
$$

and set

$$
h(t)=p(t)-1
$$

Then, we have that $h(0)=-1<0$ and $h(t) \rightarrow+\infty$ as $t \rightarrow r_{0}^{-}$. Hence, function $h$ has a smallest zero $r_{p} \in\left(0, r_{0}\right)$. Furthermore, define functions on the interval $\left[0, r_{0}\right)$ by

$$
\begin{aligned}
p_{1}(t) & =\frac{2 M_{0} M t^{2}}{1-\left(1+\frac{M_{0}}{2}\right) L_{0} t}, \\
g_{2}(t) & \left.=\frac{1}{2\left(1-L_{0} t\right)}\left[L+\frac{2 M^{2} \alpha\left(L M_{0} p_{1}(t)+2 M^{2} g_{1}(t)\right) t}{\left(1-\left(1+\frac{M_{0}}{2}\right) L_{0} t\right.}\right)(1-p(t))\right] t
\end{aligned}
$$

and set

$$
h_{2}(t)=g_{2}(t)-1
$$

Then, we have $h_{2}(0)=-1<0$ and $h_{2}(t) \rightarrow+\infty$ as $t \rightarrow r_{0}^{-}$. Hence, function $h_{2}$ has a smallest zero $r_{2} \in\left(0, r_{0}\right)$. Set

$$
r=\min \left\{r_{1}, r_{2}, r_{p}\right\}
$$

Then, we get that for each $t \in[0, r)$

$$
\begin{aligned}
& 0 \leq g_{1}(t)<1, \\
& 0 \leq p(t)<1, \\
& 0 \leq p_{1}(t)
\end{aligned}
$$

and

$$
0 \leq g_{2}(t)<1
$$

Next, using the above notation we present the local convergence analysis of method (2).

THEOREM 2.1 Let $F: D \subseteq S \rightarrow S$ be a differentiable function. Suppose that there exist $x^{*} \in D, \quad \alpha>0, L_{0}>0, L>0, M_{0}>0$ and $M>0$ such that for each $x, y \in D$ the following hold

$$
\begin{aligned}
& F\left(x^{*}\right)=0, F^{\prime}\left(x^{*}\right) \neq 0, \text { with }\left|F^{\prime}\left(x^{*}\right)\right| \mid \leq \alpha, \\
& \left|F^{\prime}\left(x^{*}\right)^{-1}\left(F^{\prime}(x)-F^{\prime}\left(x^{*}\right)\right)\right| \leq L_{0}\left|x-x^{*}\right|, \\
& \left|F^{\prime}\left(x^{*}\right)^{-1}\left(F^{\prime}(x)-F^{\prime}(y)\right)\right| \leq L|x-y|, \\
& \left|F^{\prime}(x)\right| \leq M_{0},
\end{aligned}
$$

$$
\left|F^{\prime}\left(x^{*}\right)^{-1} F^{\prime}(x)\right| \leq M
$$

and

$$
\bar{U}\left(x^{*},\left(1+M_{0}\right) r\right) \subseteq D,
$$

where $r$ is defined by (1). Then, the sequence $\left\{x_{n}\right\}$ generated by method (2) for $x_{0} \in U\left(x^{*}, r\right)-\left\{x^{*}\right\}$ is well defined, remains in $U\left(x^{*}, r\right)$ for each $n=0,1,2, \cdots$ and converges to $x^{*}$. Moreover, the following estimates hold for each $n=0,1,2, \cdots$,

$$
\left|y_{n}-x^{*}\right| \leq g_{1}\left(\left|x_{n}-x^{*}\right|\right)\left|x_{n}-x^{*}\right|<\left|x_{n}-x^{*}\right|<r,
$$

and

$$
\left|x_{n+1}-x^{*}\right| \leq g_{2}\left(\left|x_{n}-x^{*}\right|\right)\left|x_{n}-x^{*}\right|<\left|x_{n}-x^{*}\right|,
$$


where the " $g$ " functions are defined above Theorem 2.1.

Furthermore, if that there exists $T \in\left[r, \frac{2}{L_{0}}\right)$ such that $\bar{U}\left(x^{*}, T\right) \subset D$, then the limit point $x^{*}$ is the only solution of equation $F(x)=0$ in $\bar{U}\left(x^{*}, T\right)$.

Proof. We shall use induction to show estimates (12) and (13). Using the hypothesis $x_{0} \in U\left(x^{*}, r\right)-\left\{x^{*}\right\}$, the definition of $r$ and (7) we get that

$\left|F^{\prime}\left(x^{*}\right)^{-1}\left(F^{\prime}\left(x_{0}\right)-F^{\prime}\left(x^{*}\right)\right)\right| \leq L_{0}\left|x_{0}-x^{*}\right|<L_{0} r<1$.

It follows from (14) and the Banach Lemma on invertible functions $[3,4,19,20,22,23]$ that $F^{\prime}\left(x_{0}\right)$ is invertible and

$$
\left|F^{\prime}\left(x_{0}\right)^{-1} F^{\prime}\left(x^{*}\right)\right| \leq \frac{1}{1-L_{0}\left|x_{0}-x^{*}\right|}<\frac{1}{1-L_{0} r} .
$$

We can write by (6) that

$$
F\left(x_{0}\right)=F\left(x_{0}\right)-F\left(x^{*}\right)=\int_{0}^{1} F^{\prime}\left(x^{*}+\theta\left(x_{0}-x^{*}\right)\left(x_{0}-x^{*}\right) d \theta .\right.
$$

Then, we have by (9), (10) and (16) that

$$
\begin{aligned}
& \left|F\left(x_{0}\right)\right| \leq \mid \int_{0}^{1} F^{\prime}\left(x^{*}+\theta\left(x_{0}-x^{*}\right)\left(x_{0}-x^{*}\right) d \theta \mid\right. \\
& \leq M_{0}\left|x_{0}-x^{*}\right|
\end{aligned}
$$

and

$$
\begin{aligned}
& \left|F^{\prime}\left(x^{*}\right)^{-1} F\left(x_{0}\right)\right| \leq \mid \int_{0}^{1} F^{\prime}\left(x^{*}\right)^{-1} F^{\prime}\left(x^{*}+\theta\left(x_{0}-x^{*}\right)\left(x_{0}-x^{*}\right) d \theta \mid\right. \\
& \leq M\left|x_{0}-x^{*}\right|
\end{aligned}
$$

where we used $\left|x^{*}+\theta\left(x_{0}-x^{*}\right)-x^{*}\right|=\theta\left|x_{0}-x^{*}\right|<r$ for each $\theta \in[0,1]$. We also have by (17) and (11) that

$$
\begin{aligned}
& \left|x_{0} \pm F\left(x_{0}\right)-x^{*}\right| \leq\left|x_{0}-x^{*}\right|+\left|F\left(x_{0}\right)\right| \\
& \leq\left|x_{0}-x^{*}\right|+M_{0}\left|x_{0}-x^{*}\right|<\left(1+M_{0}\right) r,
\end{aligned}
$$

so $x_{0} \pm F\left(x_{0}\right) \in D$. Next we shall show that $F\left(x_{0}+F\left(x_{0}\right)\right)-F\left(x_{0}-F\left(x_{0}\right)\right)$ is invertible. Using the definition of $r_{0},(7)$ and (17), we get in turn that

$$
\begin{aligned}
& \left|F^{\prime}\left(x^{*}\right)^{-1}\left[F\left(x_{0}+F\left(x_{0}\right)\right)-F\left(x_{0}-F\left(x_{0}\right)\right)-F^{\prime}\left(x^{*}\right)\right]\right| \\
& =\mid \int_{0}^{1}\left[F^{\prime}\left(x^{*}\right)^{-1}\left[F^{\prime}\left(x_{0}-F\left(x_{0}\right)+2 \theta F\left(x_{0}\right)\right)-F^{\prime}\left(x^{*}\right)\right] d \theta \mid\right.
\end{aligned}
$$

$$
\begin{aligned}
& \leq L_{0}\left[\left|x_{0}-x^{*}\right|+\int_{0}^{1}\left|1-2 \theta \| F\left(x_{0}\right)\right| d \theta\right] \\
& \leq L_{0}\left[\left|x_{0}-x^{*}\right|+\frac{M_{0}}{2}\left|x_{0}-x^{*}\right|\right] \\
& =L_{0}\left(1+\frac{M_{0}}{2}\right)\left|x_{0}-x^{*}\right|<L_{0}\left(1+\frac{M_{0}}{2}\right) r_{0}<1 .
\end{aligned}
$$

It follows from (19) that $F\left(x_{0}+F\left(x_{0}\right)\right)-F\left(x_{0}-F\left(x_{0}\right)\right.$ is invertible and

$$
\begin{aligned}
& \mid\left(F\left(x_{0}+F\left(x_{0}\right)\right)-F\left(x_{0}-F\left(x_{0}\right)\right)^{-1} F^{\prime}\left(x^{*}\right) \mid \leq \frac{1}{1-L_{0}\left(1+\frac{M_{0}}{2}\right)\left|x_{0}-x^{*}\right|}\right. \\
& \quad<\frac{1}{L_{0}\left(1+\frac{M_{0}}{2}\right) r} .
\end{aligned}
$$

Hence, $y_{0}$ is well defined by the first substep of method (2) for $n=0$. Then, we can write

$$
\begin{gathered}
y_{0}-x^{*}=x_{0}-x^{*}-\frac{F\left(x_{0}\right)}{F^{\prime}\left(x_{0}\right)}+\frac{F\left(x_{0}\right)}{F^{\prime}\left(x_{0}\right)}-\frac{2 F\left(x_{0}\right)}{F\left(x_{0}+F\left(x_{0}\right)\right)-F\left(x_{0}-F\left(x_{0}\right)\right)} \\
=-\left[F^{\prime}\left(x_{0}\right)^{-1} F^{\prime}\left(x^{*}\right)\right]\left[\int_{0}^{1} F^{\prime}\left(x^{*}\right)^{-1}\left[F\left(x^{*}+\theta\left(x_{0}-x^{*}\right)\right)-F^{\prime}\left(x_{0}\right)\right]\right.
\end{gathered}
$$$$
\left.\times\left(x_{0}-x^{*}\right) d \theta\right]+\frac{\Gamma}{\Gamma_{1}}
$$

where

$\Gamma:=2\left(F^{\prime}\left(x^{*}\right)^{-1} F^{\prime}\left(x_{0}\right)\right)^{2}\left[\int_{0}^{1} F^{\prime}\left(x^{*}\right)^{-1}\left(F^{\prime}\left(x_{0}-F\left(x_{0}\right)+2 \theta F\left(x_{0}\right)\right)-F^{\prime}\left(x_{0}\right)\right] F^{\prime}\left(x^{*}\right) d \theta\right.$ and

$$
\Gamma_{1}:=\left[F^{\prime}\left(x^{*}\right)^{-1} F^{\prime}\left(x_{0}\right)\right]\left[F^{\prime}\left(x^{*}\right)^{-1}\left(F\left(x_{0}+F\left(x_{0}\right)\right)-F\left(x_{0}-F\left(x_{0}\right)\right)\right] .\right.
$$

The first expression at the right hand side of (21), using (8) and (15) gives

$$
\begin{aligned}
& \left.\mid F^{\prime}\left(x_{0}\right)^{-1} F^{\prime}\left(x^{*}\right)\right]\left[\int_{0}^{1} F^{\prime}\left(x^{*}\right)^{-1}\left[F\left(x^{*}+\theta\left(x_{0}-x^{*}\right)\right)-F^{\prime}\left(x_{0}\right)\right]\left(x_{0}-x^{*}\right) d \theta \mid\right. \\
& \quad \leq \frac{L\left|x_{0}-x^{*}\right|}{2\left(1-L_{0}\left|x_{0}-x^{*}\right|\right)} .
\end{aligned}
$$

Using (6), (8), (17) and (18) the numerator of the second expression in (21) gives

$$
\begin{aligned}
& \mid 2\left(F^{\prime}\left(x^{*}\right)^{-1} F^{\prime}\left(x_{0}\right)\right)^{2}\left[\int_{0}^{1} F^{\prime}\left(x^{*}\right)^{-1}\left(F^{\prime}\left(x_{0}-F\left(x_{0}\right)+2 \theta F\left(x_{0}\right)\right)-F^{\prime}\left(x_{0}\right)\right] F^{\prime}\left(x^{*}\right) d \theta \mid\right. \\
& \quad \leq 2 \alpha M^{2}\left|x_{0}-x^{*}\right|^{2} L \int_{0}^{1}|1-2 \theta| d \theta\left|F\left(x_{0}\right)\right| \\
& \quad \leq M^{2} M_{0} \alpha L\left|x_{0}-x^{*}\right|^{3} .
\end{aligned}
$$


Then, it follows from (2), (15), (20), (21)-(23) that

$$
\begin{aligned}
& \left|y_{0}-x^{*}\right| \leq \frac{L\left|x_{0}-x^{*}\right|^{2}}{2\left(1-L_{0}\left|x_{0}-x^{*}\right|\right)} \\
& +\frac{2 \alpha L M_{0} M^{2}\left|x_{0}-x^{*}\right|^{3}}{2\left(1-L_{0}\left|x_{0}-x^{*}\right|\right)\left(1-\left(1+\frac{M_{0}}{2}\right) L_{0}\left|x_{0}-x^{*}\right|\right)} \\
& =g_{1}\left(\left|x_{0}-x^{*}\right|\right)\left|x_{0}-x^{*}\right|<\left|x_{0}-x^{*}\right|<r,
\end{aligned}
$$

which shows (12) for $n=0$ and $y_{0} \in U\left(x^{*}, r\right)$. Next, we shall show that $2 F\left(y_{0}\right)-F\left(x_{0}\right)$ is invertible. First notice that by the first substep of method (2) for $n=0,(9),(10),(20)$ and the definition of function $p_{1}$ we have that

$$
\begin{aligned}
& \left|y_{0}-x_{0}\right|=2\left|\frac{F^{\prime}\left(x^{*}\right)^{-1} F\left(x_{0}\right) F\left(x_{0}\right)}{F^{\prime}\left(x^{*}\right)^{-1}\left(F\left(x_{0}+F\left(x_{0}\right)\right)-F\left(x_{0}-F\left(x_{0}\right)\right)\right.}\right| \\
& \leq \frac{2 M_{0} M\left|x_{0}-x^{*}\right|^{2}}{1-\left(1-\left(1+\frac{M_{0}}{2}\right) L_{0}\left|x_{0}-x^{*}\right|\right)}=p_{1}\left(\left|x_{0}-x^{*}\right|\right) .
\end{aligned}
$$

Then, using the definition of function $p, x_{0} \neq x^{*},(3),(4),(7),(12)$ (for $n=0$ ) and (24), we get in turn that

$$
\begin{aligned}
& \mid\left(F^{\prime}\left(x^{*}\right)\left(x_{0}-x^{*}\right)^{-1}\left[2 F\left(y_{0}\right)-F\left(x_{0}\right)-F^{\prime}\left(x^{*}\right)\left(x_{0}-x^{*}\right)\right] \mid\right. \\
& \leq\left|x_{0}-x^{*}\right|^{-1} \mid\left[2\left|F^{\prime}\left(x^{*}\right)^{-1}\left[F\left(y_{0}\right)-F\left(x^{*}\right)-F^{\prime}\left(x^{*}\right)\left(y_{0}-x^{*}\right)\right]\right|\right. \\
& \left.+2\left|y_{0}-x_{0}\right|+\left|F^{\prime}\left(x^{*}\right)^{-1}\left[F\left(x_{0}\right)-F\left(x^{*}\right)-F^{\prime}\left(x^{*}\right)\left(x_{0}-x^{*}\right)\right]\right|\right] \\
& \leq\left|x_{0}-x^{*}\right|^{-1}\left[L_{0}\left|y_{0}-x^{*}\right|^{2}+2 p_{1}\left(\left|x_{0}-x^{*}\right|\right)\right. \\
& \left.+\frac{L_{0}}{2}\left|x_{0}-x^{*}\right|^{2}\right] \\
& \leq\left[L_{0} g_{1}^{2}\left(\left|x_{0}-x^{*}\right|\right)+\frac{4 M_{0} M}{1-\left(1-\left(1+\frac{M_{0}}{2}\right) L_{0}\left|x_{0}-x^{*}\right|\right)}+\frac{L_{0}}{2}\right]\left|x_{0}-x^{*}\right| \\
& =p\left(\left|x_{0}-x^{*}\right|\right)<1 .
\end{aligned}
$$

It follows from (25) that $2 F\left(y_{0}\right)-F\left(x_{0}\right)$ is invertible and

$$
\left|\left(2 F\left(y_{0}\right)-F\left(x_{0}\right)\right)^{-1} F^{\prime}\left(x^{*}\right)\right| \leq \frac{1}{1-p\left(\left|x_{0}-x^{*}\right|\right)} .
$$

Hence, $x_{1}$ is well defined by the second step of method (2) for $n=0$. We can also write that

$$
x_{1}-x^{*}=x_{0}-x^{*}-\frac{F\left(x_{0}\right)}{F^{\prime}\left(x_{0}\right)}+\frac{F\left(x_{0}\right)}{F^{\prime}\left(x_{0}\right)}-\frac{2 F\left(x_{0}\right)^{2}}{F\left(x_{0}+F\left(x_{0}\right)\right)-F\left(x_{0}-F\left(x_{0}\right)\right)}
$$

$$
\begin{aligned}
& \times \frac{F\left(y_{0}\right)-F\left(x_{0}\right)}{2 F\left(y_{0}\right)-F\left(x_{0}\right)} \\
& =x_{0}-x^{*}-\frac{F\left(x_{0}\right)}{F^{\prime}\left(x_{0}\right)} \\
& +\frac{N}{\Gamma_{2}}
\end{aligned}
$$

where

$F^{\prime}\left(x^{*}\right)^{4} N=F\left(x_{0}\right)\left(2 F\left(y_{0}\right)-F\left(x_{0}\right)\right)\left(F\left(x_{0}+F\left(x_{0}\right)\right)-F\left(x_{0}-F\left(x_{0}\right)\right)\right.$

$\left.-2 F\left(x_{0}\right) F^{\prime}\left(x_{0}\right)\left(F\left(y_{0}\right)-F\left(x_{0}\right)\right)\right)$

$=2 F\left(x_{0}\right)^{2}\left\{\int_{0}^{1}\left[F^{\prime}\left(x_{0}-F\left(x_{0}\right)+2 \theta F\left(x_{0}\right)\right)-F^{\prime}\left(x_{0}\right)\right]\left(F\left(y_{0}\right)-F\left(x_{0}\right)\right) d \theta\right.$

$\left.+\int_{0}^{1} F^{\prime}\left(x_{0}-F\left(x_{0}\right)+2 \theta F\left(x_{0}\right)\right) F\left(y_{0}\right) d \theta\right\}$

and

$\Gamma_{2}:=\left(F^{\prime}\left(x^{*}\right)^{-1} F^{\prime}\left(x_{0}\right)\right) F^{\prime}\left(x^{*}\right)^{-1}\left(F\left(x_{0}+F\left(x_{0}\right)\right)-F\left(x_{0}-F\left(x_{0}\right)\right) F^{\prime}\left(x^{*}\right)^{-1}\left(2 F\left(y_{0}\right)-F\left(x_{0}\right)\right)\right.$.

Using (9), (17), (18), (24) and (28), we get that

$|N| \leq 2\left|F^{\prime}\left(x^{*}\right) \| F^{\prime}\left(x^{*}\right)^{-1} F\left(x_{0}\right)\right|^{2}\left\{\mid \int_{0}^{1} F^{\prime}\left(x^{*}\right)^{-1}\left[F^{\prime}\left(x_{0}-F\left(x_{0}\right)\right.\right.\right.$

$\left.\left.+2 \theta F\left(x_{0}\right)\right)-F^{\prime}\left(x_{0}\right)\right] d \theta$

$\times\left|\int_{0}^{1} F^{\prime}\left(x^{*}\right)^{-1} F^{\prime}\left(x_{0}-F\left(x_{0}\right)+2 \theta F\left(x_{0}\right)\right)\left(y_{0}-x_{0}\right) d \theta\right|$

$\left.+\int_{0}^{1} F^{\prime}\left(x^{*}\right)^{-1} F^{\prime}\left(x_{0}-F\left(x_{0}\right)+2 \theta F\left(x_{0}\right)\right) d \theta \| F^{\prime}\left(x^{*}\right)^{-1} F\left(y_{0}\right)\right\}$

$\leq 2 \alpha M^{2}\left|x_{0}-x^{*}\right|^{2}\left[\frac{L M_{0}}{2}\left|x_{0}-x^{*}\right|\left|y_{0}-x^{*}\right|+M^{2}\left|y_{0}-x^{*}\right|\right]$

$\leq \alpha M^{2}\left|x_{0}-x^{*}\right|^{2}\left[L M_{0}\left|x_{0}-x^{*}\right| p_{1}\left(\left|x_{0}-x^{*}\right|\right)+2 M^{2} g_{1}\left(\left|x_{0}-x^{*}\right|\right)\left|x_{0}-x^{*}\right|\right]$

$\leq \alpha M^{2}\left(L M_{0} p_{1}\left(\left|x_{0}-x^{*}\right|\right)+2 M^{2} g_{1}\left(\left|x_{0}-x^{*}\right|\right)\right)\left|x_{0}-x^{*}\right|^{3}$.

Then, using (5), (15), (20), (22) and (26)-(30), we get that

$$
\begin{aligned}
& \left|x_{1}-x^{*}\right| \leq \frac{L\left|x_{0}-x^{*}\right|^{2}}{2\left(1-L_{0}\left|x_{0}-x^{*}\right|\right)} \\
& +\frac{2 \alpha M^{2}\left[L M_{0} p_{1}\left(\left|x_{0}-x^{*}\right|\right)+2 M^{2} g_{1}\left(\left|x_{0}-x^{*}\right|\right)\right]\left|x_{0}-x^{*}\right|^{3}}{2\left(1-L_{0}\left|x_{0}-x^{*}\right|\right)\left(1-\left(1+\frac{M_{0}}{2}\right) L_{0}\left|x_{0}-x^{*}\right|\right)\left(1-p\left(\left|x_{0}-x^{*}\right|\right)\right)} \\
& =g_{2}\left(\left|x_{0}-x^{*}\right|\right)\left|x_{0}-x^{*}\right|<\left|x_{0}-x^{*}\right|<r,
\end{aligned}
$$

which shows (13) for $n=0$ and $x_{1} \in U\left(x^{*}, r\right)$. By simply replacing 
$x_{0}, y_{0}, x_{1}$ by $x_{k}, y_{k}, x_{k+1}$ in the preceding estimates we arrive at estimates (12) and (13). Using the estimate $\left|x_{k+1}-x^{*}\right|<\left|x_{k}-x^{*}\right|<r$, we deduce that $x_{k+1} \in U\left(x^{*}, r\right)$ and $\lim _{k \rightarrow \infty} x_{k}=x^{*}$. To show the uniqueness part, let $Q=\int_{0}^{1} F^{\prime}\left(y^{*}+\theta\left(x^{*}-y^{*}\right) d \theta\right.$ for some $y^{*} \in \bar{U}\left(x^{*}, T\right)$ with $F\left(y^{*}\right)=0$. Using (6) we get that

$$
\begin{aligned}
& \left|F^{\prime}\left(x^{*}\right)^{-1}\left(Q-F^{\prime}\left(x^{*}\right)\right)\right| \leq \int_{0}^{1} L_{0}\left|y^{*}+\theta\left(x^{*}-y^{*}\right)-x^{*}\right| d \theta \\
& \leq \int_{0}^{1}(1-\theta)\left|x^{*}-y^{*}\right| d \theta \leq \frac{L_{0}}{2} R<1 .
\end{aligned}
$$

It follows from (30) and the Banach Lemma on invertible functions that $Q$ is invertible. Finally, from the identity $0=F\left(x^{*}\right)-F\left(y^{*}\right)=Q\left(x^{*}-y^{*}\right)$, we deduce that $x^{*}=y^{*}$.

\section{REMARK 2.2}

1. In view of (8) and the estimate

$\left\|F^{\prime}\left(x^{*}\right)^{-1} F^{\prime}(x)\right\|=\left\|F^{\prime}\left(x^{*}\right)^{-1}\left(F^{\prime}(x)-F^{\prime}\left(x^{*}\right)\right)+I\right\|$

$\leq 1+\left\|F^{\prime}\left(x^{*}\right)^{-1}\left(F^{\prime}(x)-F^{\prime}\left(x^{*}\right)\right)\right\| \leq 1+L_{0}\left\|x-x^{*}\right\|$

condition (10) can be dropped and $M$ can be replaced by

$M(t)=1+L_{0} t$.

2. The results obtained here can be used for operators $F$ satisfying autonomous differential equations [3] of the form

$F^{\prime}(x)=P(F(x))$

where $P$ is a continuous operator. Then, since $F^{\prime}\left(x^{*}\right)=P\left(F\left(x^{*}\right)\right)=P(0)$, we can apply the results without actually knowing $x^{*}$. For example, let $F(x)=e^{x}-1$. Then, we can choose: $P(x)=x+1$.

3. The radius $r_{A}$ was shown by us to be the convergence radius of Newton's method [2]-[4]

$$
x_{n+1}=x_{n}-F^{\prime}\left(x_{n}\right)^{-1} F\left(x_{n}\right) \text { foreach } n=0,1,2, \cdots
$$

under the conditions (8) and (9). It follows from the definition of $r$ that the convergence radius $r$ of the method (2) cannot be larger than the convergence radius $r_{A}$ of the second order Newton's method (31) if $L_{0} M_{0} \geq L$. Even in the case $L_{0} M_{0}<L$, still $r$ may be smaller than $r_{A}$. As already noted in [3, 4] $r_{A}$ is at least as large as the convergence ball given by Rheinboldt [25]

$r_{R}=\frac{2}{3 L}$.

In particular, for $L_{0}<L$ we have that

$$
\begin{aligned}
& r_{R}<r \\
& \text { and } \\
& \frac{r_{R}}{r_{A}} \rightarrow \frac{1}{3} \text { as } \frac{L_{0}}{L} \rightarrow 0 .
\end{aligned}
$$

and

That is our convergence ball $r_{A}$ is at most three times larger than Rheinboldt's. The same value for $r_{R}$ was given by Traub [26].

4. It is worth noticing that method (2) is not changing when we use the conditions of Theorem 2.1 instead of the stronger conditions used in $[1,5,11-28]$. Moreover, we can compute the computational order of convergence (COC) defined by

$$
\xi=\ln \left(\frac{\left\|x_{n+1}-x^{*}\right\|}{\left\|x_{n}-x^{*}\right\|}\right) / \ln \left(\frac{\left\|x_{n}-x^{*}\right\|}{\left\|x_{n-1}-x^{*}\right\|}\right)
$$

or the approximate computational order of convergence

$$
\xi_{1}=\ln \left(\frac{\left\|x_{n+1}-x_{n}\right\|}{\left\|x_{n}-x_{n-1}\right\|}\right) / \ln \left(\frac{\left\|x_{n}-x_{n-1}\right\|}{\left\|x_{n-1}-x_{n-2}\right\|}\right) .
$$

This way we obtain in practice the order of convergence in a way that avoids the bounds involving estimates using estimates higher than the first Fréchet derivative of operator $F$.

\section{NUMERICAL EXAMPLES}

We present numerical examples in this section.

EXAMPLE 3.1 Let $D=[-\infty,+\infty]$. Define function $f$ of $D$ by

$$
f(x)=\sin (x) .
$$

Then we have for $x^{*}=0$ that $L_{0}=L=M=M_{0}=1, \alpha=1$. The parameters are given in Table 1.

\begin{tabular}{|c|}
\hline$r_{A}=0.6667$ \\
\hline$r_{0}=0.6667$ \\
\hline$r_{1}=0.4000$ \\
\hline$r_{p}=0.1138$ \\
\hline$r_{2}=0.2240$ \\
$\xi_{1}=4.9901$ \\
\hline
\end{tabular}

Table 1

EXAMPLE 3.2 Let $D=[-1,1]$. Define function $f$ of $D$ by

$$
f(x)=e^{x}-1 .
$$

Using (2) and $x^{*}=0$, we get that $L_{0}=e-1<L=M=M_{0}=e, \alpha=1$. The parameters are given in Table 2 . 


\begin{tabular}{|l|}
\hline$r_{A}=0.3249$ \\
\hline$r_{0}=0.2467$ \\
\hline$r_{1}=0.0967$ \\
\hline$r_{p}=0.0262$ \\
\hline$r_{2}=0.0372$ \\
\hline$\xi_{1}=4.3370$ \\
\hline
\end{tabular}

Table 2

EXAMPLE 3.3 Returning back to the motivational example at the introduction of this study, we have $L_{0}=L=146.6629073, M=101.5578008, M_{0}=3 M, \alpha=1$. parameters are given in Table 3.

\begin{tabular}{|c|}
\hline$r_{A}=0.0045$ \\
\hline$r_{0}=4.4467 e-\sigma$ \\
\hline$r_{1}=0.2818$ \\
\hline$r_{p}=0.0575$ \\
\hline$r_{2}=0.0001$ \\
\hline$\xi_{1}=3.8283$ \\
\hline
\end{tabular}

Table 3

\section{REFERENCES}

[1] S. Amat, M.A. Hernández, N. Romero, A modified Chebyshev's iterative method with at least sixth order of convergence, Appl. Math. Comput. 206(1), 164-174 (2008).

[2] S. Amat, S. Busquier, S. Plaza, Dynamics of the King's and Jarratt iterations, Aequationes. Math. 69, (2005), 212-213.

[3] I.K. Argyros, "Convergence and Application of Newton-type Iterations," Springer, 2008.

[4] I. K. Argyros and Said Hilout, Computational methods in nonlinear Analysis, World Scientific Publ. Co. , New Jersey, USA, 2013.

[5] I. K. Argyros D.Chen, Q. Quian, The Jarratt method in Banach space setting, J.Comput.Appl.Math. 51,(1994), 103-106.

[6] V. Candela, A. Marquina, Recurrence relations for rational cubic methods I: The Halley method, Computing, 44, 169-184(1990).

[7] J.Chen, Some new iterative methods with three-order convergence, Appl. Math. Comput. 181, (2006), 1519-1522.

[8] C.Chun, B. Neta, M. Scott, Basins of attraction for optimal eighth order methods to find simple roots of nonlinear equations, Appl. Math. Comput. 227, (2014), 567-592.

[9] A. Cordero, J. Torregrosa, Variants of Newton's method using fifth order quadrature formulas, Appl.Math.Comput. 190,(2007), 686-698.

[10] A. Cordero, J. Maimo, J. Torregrosa, M.P. Vassileva, P. Vindel, Chaos in King's iterative family, Appl. Math. Lett. 26, (2013), 842-848.

[11] A. Cordero, J. L. Hueso, E. Martinez, J.R. Torregrossa, Steffensen type methods for solving non-linear equations, J. Comput. Appl. Math. 236, (2012), 3058-3064.

[12] J. A. Ezquerro, M.A. Hernández, A uniparametric Halley-type iteration with free second derivative, Int. J.Pure and Appl. Math. 6, 1, (2003), 99110 .
[13] J. A. Ezquerro, M.A. Hernández, New iterations of R-order four with reduced computational cost. BIT Numer. Math. 49, 325- 342 (2009).

[14] M. Frontini, E. Sormani, Some variants of Newton's method with third order convergence, Appl. Math. Comput. 140, (2003), 419-426.

[15] J.M. Gutiérrez, M.A. Hernández, Recurrence relations for the superHalley method, Computers Math. Applic. 36(7), 1-8(1998).

[16] M.A. Hernández, M.A. Salanova, Sufficient conditions for semilocal convergence of a fourth order multipoint iterative method for solving equations in Banach spaces. Southwest J. Pure Appl. Math(1), 2940(1999).

[17] R.F. King, A family of fourth-order methods for nonlinear equations, SIAM. J. Numer. Anal. 10, (1973), 876-879.

[18] A. A. Magreñán, Different anomalies in a Jarratt family of iterative rootfinding methods, Applied Mathematics and Computation, 233, p.29-38.

[19] A. K. Maheshwari, A fourth order iterative method for solving nonlinear equations, Appl. Math. Comput. 211, (2009), 283-391.

[20] S. K. Parhi, D.K. Gupta, Semi-local convergence of a Stirling-like method in Banach spaces, Int. J. Comput. Methods 7(02), 215-228(2010).

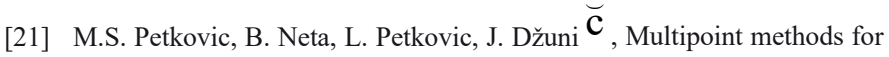
solving nonlinear equations, Elsevier, 2013.

[22] F.A. Potra, V. Ptak, Nondiscrete induction and iterative processes, Research Notes in Mathematics, Vol. 103, Pitman Publ., Boston, MA, 1984.

[23] L. B. Rall, Computational solution of nonlinear operator equations, Robert E. Krieger, New York(1979).

[24] H. Ren, Q. Wu, W. Bi, New variants of Jarratt method with sixth-order convergence, Numer. Algorithms 52(4), 585-603(2009).

[25] W.C. Rheinboldt,An adaptive continuation process for solving systems of nonlinear equations, In: Mathematical models and numerical methods (A.N.Tikhonov et al. eds.) pub.3, (19), 129-142 Banach Center, Warsaw Poland.

[26] J.F.Traub, Iterative methods for the solution of equations, Prentice Hall Englewood Cliffs, New Jersey, USA, 1964.

[27] S. Weerakoon, T.G.I. Fernando, A variant of Newton's method with accelerated third-order convergence, Appl. Math. Lett. 13, (2000), 87-93.

[28] X. Wang, J. Kou, Convergence for modified Halley-like methods with less computation of inversion, J. Diff. Eq. and Appl. 19, 9, (2013), 1483-1500.

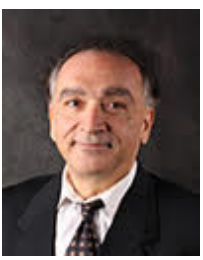

Ioannis K. Argyros was born in 1956 in Athens, Greece. $\mathrm{He}$ received a B.Sc from the University of Athens, Greece; and a M.Sc and Ph.D from the Univer-sity of Georgia, Athens, Georgia, USA, under the supervision of Dr. Douglas N. Clark. Dr. Argyros is currently a full professor of Mathematics at Cameron Uni-versity, Lawton, OK, USA. He published more than 800 papers and 17 books/ monographs in his area of research, computational mathematics. He is also the editor of 20 peer reviewed research journals in Mathematics.

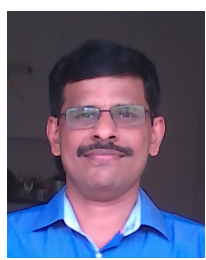

Santhosh George was born in 1967 in Kerala, India. $\mathrm{He}$ received his $\mathrm{PhD}$ degree in Mathematics from Goa University, under the supervision of Dr. M. T. Nair. Dr. George is a Professor of Mathematics at National Institute of Technology, Karnataka. Five students have completed their $\mathrm{PhD}$ under his guidance. He has many International journal and conference papers to his credit 